\title{
Long non-coding RNA LINC01224 promotes cell proliferation and inhibits apoptosis by regulating AKT3 expression via targeting miR-485-5p in endometrial carcinoma
}

\author{
XIN ZUO*, WEILING LI* ${ }^{*}$ XIAOFANG YAN* ${ }^{*}$ TIELIANG MA, YAN REN, \\ MEIJUAN HUA, HUIYUN YANG, HAIFENG WU and HONGDI ZHU \\ Department of Obstetrics and Gynecology, The Affiliated Yixing Hospital of \\ Jiangsu University, Yixing, Jiangsu 214200, P.R. China
}

Received August 5, 2020; Accepted May 20, 2021

DOI: 10.3892/or.2021.8137

\begin{abstract}
Endometrial carcinoma (EC) is the most common cancer in women worldwide, yet little is known about the underlying molecular basis of EC development. LINC01224, a novel long non-coding (lnc)RNA, was recently identified as an oncogene in various types of cancer. However, the function and underlying mechanism of LINC01224 in EC is still unclear. A total of 50 pairs of tumor and adjacent normal tissue from patients with EC, three EC cell lines and one human normal endometrial stromal cell (ESC) line were subjected to reverse transcription-quantitative PCR assay to evaluate the expression levels of LINC01224. Cell Counting Kit-8, colony formation and flow cytometry assays were used to assess cell proliferation and apoptosis. Western blotting was used to measure expression levels of apoptosisand proliferation-associated proteins and AKT3 protein. A xenograft model of HEC1A cells was established to validate the in vivo function of LINC01224 in EC tumor growth. Starbase 3.0 database prediction and luciferase reporter and RNA pull-down assays were performed to verify the binding sites between LINC01224 and microRNA (miR)-485-5p
\end{abstract}

Correspondence to: Mr. Hongdi Zhu, Department of Obstetrics and Gynecology, The Affiliated Yixing Hospital of Jiangsu University, 75 Tongzhenguan Road, Yixing, Jiangsu 214200, P.R. China

E-mail: hongdizhu_1964@163.com

*Contributed equally

Abbreviations: CCK-8, Cell Counting Kit-8; EC, Endometrial carcinoma; miRNA, microRNA; MT, mutant; Con, Control; RT-q, reverse transcription-quantitative; GTEx, Genotype Tissue Expression; NC, negative control; shRNA, short hairpin RNA; TCGA, The Cancer Genome Atlas; UTR untranslated region; WT, wild-type

Key words: endometrial carcinoma, long non-coding RNA, LINC01224, proliferation, apoptosis, miRNA-485-5p, AKT3 and miR-485-5p and AKT3. LINC01224 expression was significantly upregulated in both EC tumor tissue and cell lines. The upregulation of LINC01224 was negatively associated with survival of patients with EC. Functionally, LINC01224 promoted proliferation and inhibited apoptosis of EC cells; LINC01224 directly bound to and downregulated miR-485-5p to elevate the expression levels of AKT3, thereby promoting EC progression. LINC01224 depletion in EC cells hindered tumor growth in a xenograft model. The tumor suppressing effect of LINC01224-knockdown on EC progression was partly rescued by treatment with miR-485-5p inhibitor. The present data demonstrated the expression levels, clinical relevance and functional mechanism of LINC01224 in EC. LINC01224 promoted EC development via sponging miR-485-5p to elevate AKT3 expression levels; this may provide a promising therapeutic target pathway for EC treatment.

\section{Introduction}

Endometrial carcinoma (EC) is the sixth most common malignancy of the female genital tract worldwide, with 380,000 new cases in 2018 (1-3). Despite improvements in EC diagnosis and treatment, the prognosis and survival rate of patients with EC is still unsatisfactory due to invasion and recurrence, with $>89,000$ deaths in $2018(2,3)$. Therefore, it is necessary to discover the underlying mechanism of the tumorigenesis of EC to develop novel biomarkers and improve therapy for patients with EC.

Long non-coding (lnc)RNAs are a group of ncRNAs $>200$ nucleotides in length that affect key biological processes, including transcriptional regulation, protein translation and degradation $(4,5)$. Novel lncRNAs have been discovered and identified as crucial regulators involved in neoplastic tumorigenesis and development, such as tumor promoter lncRNA Xist, lncRNA HOTAIR and tumor suppressor lncRNA MEG3, TDRG1 (6-10), offering the possibility of lncRNAs as effective biomarkers and therapeutic targets for cancer. However, the association between the deregulation of lncRNAs and carcinogenesis remains unknown $(5,11)$. Thus, identification of new lncRNAs not only provides insight into their functional roles 
but may also elucidate the potential mechanism of lncRNAs in human cancer, including EC.

Recently, LINC01224, a cancer-associated lncRNA, has been identified to be involved in carcinogenesis and to promote tumor proliferation, invasion, migration and apoptosis; its upregulation is associated with tumor grade and poor prognosis in epithelial ovarian cancer and hepatocellular carcinoma $(12,13)$. However, the role and underlying molecular mechanism of LINC01224 in EC remains unclear. MicroRNAs (miRNAs/miRs) are short non-protein-coding RNAs $\sim 22$ nucleotides in length that inhibit gene expression by targeting the 3'-untranslated region (UTR) of mRNA. Accumulating evidence has demonstrated that miRNAs serve crucial roles in multiple biological processes, including cell growth, differentiation and apoptosis $(14,15)$. IncRNAs serve as competing endogenous RNAs by sponging miRNAs, thus restoring miRNA-induced functions of target genes $(6,16)$. Moreover, it has been reported that LINC01224 promotes epithelial ovarian cancer progression via sponging certain miRNAs and LINC01224 inhibits hepatocellular carcinoma progression via sponging miR-330-5p $(12,13)$. However, whether LINC01224 interacts with miRNA in EC remains to be determined. Therefore, discovering the novel lncRNA-miRNA axis in EC is urgent for the identification of promising diagnostic biomarkers and therapeutic targets for EC treatment.

LINC01224 expression levels in EC tumor tissue and cell lines, and its association with survival of patients with EC, were assessed. The phenotypes of LINC01244 knockdown both in vitro and in vivo were also determined. The present study aimed to investigate the functional mechanism and clinical significance of LINC01224 in EC.

\section{Materials and methods}

Clinical patient samples. A total of 50 pairs of EC and adjacent (distance, $\geq 3 \mathrm{~cm}$ ) para-carcinoma tissue samples were obtained from the Affiliated Yixing Hospital of Jiangsu University (Yixing, China). A total of 50 female patients (age, 42-68 years) were enrolled from January 2017 to January 2019. Patients were diagnosed with primary EC by two pathologists and had not received any therapy before sample collection. The tissues were instantly placed into liquid nitrogen to protect RNA integrity and stored at $-80^{\circ} \mathrm{C}$ for further use. All patient sample collections and experiments were approved by the ethics committee of the Affiliated Yixing Hospital of Jiangsu University and performed in accordance with the guidelines of the Declaration of Helsinki. Written informed consent was obtained from all patients.

Cell lines. The human endometrial stromal cell (ESC) line, CL0453, was provided by Cell Bank of Chinese Academy of Sciences (cat. no. BNCC267006; Shanghai, China). Three EC cell lines (HEC1A, HEC1B and Ishikawa) and 293T cells were purchased from American Type Culture Collection (ATCC). Human ESC cells were cultured according to the protocol of the Cell Bank of Chinese Academy of Sciences [1:1 DMEM and Ham's F-12 medium (both Gibco; Thermo Fisher Scientific, Inc.) containing $1.2 \mathrm{~g} / 1$ sodium bicarbonate, $2.5 \mathrm{mM}$ L-glutamine, $15 \mathrm{mM}$ HEPES and $0.5 \mathrm{mM}$ sodium pyruvate supplemented with $2.0 \mathrm{mM}$ L-Alanyl-L-Glutamine, $0.1 \mathrm{mM}$ non-essential amino acids, $0.1 \mathrm{mM} 2$-mercaptoethanol and $4 \mathrm{ng} / \mathrm{ml}$ basic fibroblastic growth factor; 5\% knockout serum replacement (Gibco; Thermo Fisher Scientific, Inc.) and $15 \%$ fetal bovine serum]; HEC1A cell line was cultured in McCoy's 5A medium (Gibco; Thermo Fisher Scientific, Inc.); 293T, HEC1B and Ishikawa cell lines were cultured in DMEM (Gibco; Thermo Fisher Scientific, Inc.) supplemented with 10\% (v/v) FBS (Gibco; Thermo Fisher Scientific, Inc.). All cell lines were cultured in a humidified $37^{\circ} \mathrm{C}$ incubator with $5 \% \mathrm{CO}_{2}$.

Cell infection. To knock down the expression of LINC01244 in EC cells, a lentiviral short hairpin (sh)RNA vector targeting shLINC01244 was generated by inserting double-stranded oligonucleotides (shLINC01244\#1, 5'-GGT TGTTGCTTCCTAGTCTGG-3'; shLINC01244\#2, 5'-GCT TCCTAGTCTGGTGGTGAA-3') into pLKO-puro Vector (Thermo Fisher Scientific, Inc.) plasmids (pLKO.1-shRNA, $1 \mu \mathrm{g}$; pCMV- $\Delta \mathrm{R} 8.2,1 \mu \mathrm{g}$; pM2D.G, $0.5 \mu \mathrm{g} /$ per well in 6 -well plates). Specific LINC01244 shRNA lentivirus was infected with HEC1A and Ishikawa cell lines for $48 \mathrm{~h}$ (Shanghai GenePharma Co., Ltd.). A total of $1 \times 10^{5}$ HEC1A and Ishikawa cells were seeded in 6-well plates and incubated for $24 \mathrm{~h}$ at $37^{\circ} \mathrm{C}$ and $5 \% \mathrm{CO}_{2}$. For the negative control (con) group, cells were added with lentivirus containing scramble shRNA (sh-con); for the LINC01244 knockdown group, cells were added with lentivirus containing shLINC01244\#1 and shLINC01244\#2. The stably infected cells were screened using puromycin $(2 \mu \mathrm{g} / \mathrm{ml})$ for 10 days.

Reverse transcription-quantitative $(R T-q) P C R$. Total RNA from HEC1A, HEC1B and Ishikawa cells was extracted following transfection using a miRNeasy Mini kit (cat. no. 217004; Qiagen GmbH). The first-strand cDNA was reverse transcribed using PrimeScript ${ }^{\mathrm{TM}}$ RT Master Mix (cat. no. RR036Q, Takara Bio, Inc.) according to manufacturer's protocol. RT-qPCR was performed using SYBR Green SuperMix (Roche Diagnostics) following the manufacturer's protocols. GAPDH and U6 were used as the internal control for IncRNA/mRNA and miRNAs, respectively. Each sample was run in triplicate using samples from independent experiments. The relative mRNA expression levels were expressed as a function of threshold cycle $(\mathrm{Cq})$ and analyzed by the $2^{-\Delta \Delta \mathrm{Cq}}$ method (17).

The primer sequences were as follows: LINC01224 forward, 5'-AGAGCTTGGGATCGCTTTCTG-3' and reverse, 5'-TTACTCAGGTGCCTTTCCCAC-3'; miR-485-5p forward, 5'-AGAGGCTGGCCGTGAT-3' and reverse, 5'-ATGTGT TGCTGTGTTTGTCG-3'; GAPDH forward, 5'-TATCGT GATGCTAGTCCGATG-3' and reverse, 5'-TGCAGCTAG CTGCATCGATCGG-3' and U6 forward, 5'-CTCGCTTCG GCAGCACA-3' and reverse, 5'-AACGCTTCACGAATTTGC GT-3'. The thermocycling conditions were as follows: $95^{\circ} \mathrm{C}$ for $15 \mathrm{~min}$, followed by $95^{\circ} \mathrm{C}$ for $30 \mathrm{sec}, 65^{\circ} \mathrm{C}$ for $30 \mathrm{sec}$ and $72^{\circ} \mathrm{C}$ for $30 \mathrm{sec}\left(35-45\right.$ cycles) and $72^{\circ} \mathrm{C}$ for $5 \mathrm{~min}$.

Cell counting kit (CCK)-8 assay. Cell proliferation was evaluated by CCK- 8 assay (Dojindo Molecular Technologies, Inc.) following the manufacturer's protocol. The transfected HEC1A 
and Ishikawa cells were seeded at a density of $2 \times 10^{3} /$ well in 96-well plates. Cells were cultured for $24,48,72$ or $96 \mathrm{~h}$ at $37^{\circ} \mathrm{C}$ with $5 \% \mathrm{CO}_{2}$. Then, $10 \mu \mathrm{lCCK}-8$ solution was added to each well and incubated for $1 \mathrm{~h}$ at $37^{\circ} \mathrm{C}$. The absorbance was measured at $450 \mathrm{~nm}$ with a microplate reader (Synergy H4 Hybrid Reader; BioTek Instruments, Inc.). Data are presented as the mean \pm SD of three independent experiments.

Colony formation. A total of 1,000-1,500 cells were seeded into 6-well plates. After culturing for 14 days in a humidified $37^{\circ} \mathrm{C}$ incubator with $5 \% \mathrm{CO}_{2}$, the colonies were fixed with $10 \%$ formaldehyde for $30 \mathrm{~min}$ at room temperature and then stained with $0.5 \%$ crystal violet (Beyotime Institute of Biotechnology) for $30 \mathrm{~min}$ at room temperature. The colonies were photographed under a light microscope at x20 magnification (Olympus Corporation).

Apoptosis assay. Transfected HEC1A and Ishikawa cells were seeded in a 6 -well plate $\left(1 \times 10^{6}\right.$ cells/well $)$. Following transfection, cells were harvested by centrifugation at $200 \mathrm{x} g$ for $5 \mathrm{~min}$ at room temperature and washed with $1 \mathrm{X}$ PBS three times, then incubated with $5 \mu 1$ FITC-conjugated Annexin V and $5 \mu \mathrm{l}$ PI $(25 \mu \mathrm{g} / \mathrm{ml})$ (both BD Biosciences) for $15 \mathrm{~min}$ at room temperature in the dark. The stained cells were detected using a BD FACSAria II flow cytometer (BD Biosciences). FlowJo V10.5.2 (BD Biosciences) software was used to analyze data.

Western blot analysis. Transfected HEC1A and Ishikawa cells were lysed in RIPA buffer (Beyotime Institute of Biotechnology). Total protein concentration was qualified with a BCA Protein Assay kit (Sangon Biotech Co., Ltd.). Next, protein samples $(\sim 1,000 \mathrm{ng} /$ lane $)$ were separated by $12 \%$ SDS-PAGE and transferred to PVDF membranes (MilliporeSigma). After blocking with 5\% non-fat milk for $1 \mathrm{~h}$ at room temperature, the membranes were incubated with primary antibodies [cleaved caspase-3, 1:500, cat. no. ab32042; Bcl-2, 1:1,000, cat. no. ab32124; proliferating cell nuclear antigen (PCNA), 1:1,000, cat. no. ab29; AKT3, 1:2,000, cat. no. ab152157; $\beta$-actin, $1: 5,000$, cat. no. ab6276] at $4^{\circ} \mathrm{C}$ overnight. Anti-rabbit (cat. no. ab150077, for cleaved caspase-3, Bcl-2, AKT3, $\beta$-actin) and anti-mouse IgG (cat. no. ab150113, 1:10,000, for PCNA) were used as the secondary antibody at room temperature for $2 \mathrm{~h}$. All the antibodies was purchased from Abcam (Shanghai, China). ECL kit (Sangon Biotech Co., Ltd.) was used for chemiluminescent detection of immobilized proteins according to the manufacturer' protocol. All antibodies were obtained from Abcam. The protein bands were quantified with ImageJ V1.8.0 software (National Institutes of Health).

Tumor xenograft experiments. A total of 12 female BALB/c nude mice (age, 4 weeks; weight, 13-15 g; Shanghai Model Organisms Center, Inc.) were used for xenograft experiments. All mice were housed in $25^{\circ} \mathrm{C}, 50 \%$ humidity and specific-pathogen-free conditions with a 12/12-h light/dark cycle. Sterile food and water were provided daily. All animal protocols were approved by the Institutional Animal Care and Use Committee at the Affiliated Yixing Hospital of Jiangsu University. Briefly, lentivirus-transfected HEC1A cells $\left(1 \times 10^{6}\right)$ were resuspended in $100 \mu 1$ DMEM (Gibco; Thermo Fisher Scientific, Inc.) without FBS and subcutaneously injected into the flank of mice. After $\sim 1$ week, the tumor was visible $\left(100 \mathrm{~mm}^{3}\right)$ and injected with sh-con, shLINC01224\#1 and shLINC01224\#2 (10 nmol/20 g) twice per week ( $n=6 /$ group). Tumor volumes were measured every 7 days. Tumor volume was calculated as follows: Tumor volume $\left(\mathrm{mm}^{3}\right)=($ height $) \mathrm{x}(\text { width })^{2} / 2$. After 28 days, mice were sacrificed for analysis and tumor weight was measured. An intraperitoneal injection of $150 \mathrm{mg} / \mathrm{kg}$ pentobarbital was used for euthanasia. The dose of pentobarbital used for anesthesia was $10 \%$ in saline, $50 \mathrm{mg} / \mathrm{kg}$, via intraperitoneal injection. Cessation of movement, breathing, and heartbeat were considered to indicate death. Mouse death was verified by cutting the chest cavity to confirm no heartbeat and touching the eyeball, which produced no reflex activity. Based on Institutional Animal Care and Use Committee guidelines, tumor size $>20 \mathrm{~mm}$ at the largest diameter was considered to be the humane endpoint (18). After 28 days, the maximum tumor weight observed was $7.8 \%$ of mouse body weight. The maximum tumor diameter observed was $17.5 \mathrm{~mm}$.

Luciferase reporter assay. The day before transfection, $293 \mathrm{~T}$ cells were plated in 24 -well plates $\left(4 \times 10^{4}\right.$ cells/well). Full-length LINC01224 gene and 3'-UTR of the AKT3 gene were cloned into pmirGLO plasmids containing luciferase (PromegaCorporation) according tothe manufacturer's protocol and transfected into $293 \mathrm{~T}$ cells. GeneArt ${ }^{\mathrm{TM}}$ Site-Directed Mutagenesis system (Thermo Fisher Scientific, Inc.) was used to produce the mutant (MUT) LINC01224 and AKT3 gene reporter. LINC01224 wild-type (WT)/MUT or AKT3 WT/MUT plasmids were co-transfected with miR-negative control (NC) or miR-485-5p mimics into 293T cells using Lipofectamine $^{\circledR} 2000$ (Invitrogen; Thermo Fisher Scientific, Inc.) according to the manufacturer's instructions for $48 \mathrm{~h}$. Following transfection for $48 \mathrm{~h}$, luciferase activity was measured immediately by Dual-Luciferase Reporter Assay system (Promega Corporation) following the manufacturer's protocol, and firefly luciferase activity was normalized against Renilla luciferase activity.

Bioinformatics analysis. Gene Expression Profiling Interactive Analysis (GEPIA2; gepia2.cancer-pku.cn/\#index) was used to analyze LINC01224 expression levels in EC. StarBase 3.0 (starbase.sysu.edu.cn/) was used to predict the interaction between LINC01224 and miRNAs and the targets of $\mathrm{miR}-485-5 \mathrm{p}$.

RNA pull-down assay. LINC01224 and NC sequence (nonsense sequence) were biotin-labeled using Biotin RNA Labeling Mix and T7/SP6 RNA polymerase (Roche Diagnostics) to create bio-LINC01224 and bio-NC by Shanghai GenePharma Co., Ltd. by using pcDNA 3.1 (GenePharma Co., Ltd., China) with T7 promoter. according to the manufacturer's protocol. Following transfection, HEC1A and Ishikawa cell lines were lysed by lysis buffer (Invitrogen; Thermo Fisher Scientific, Inc.) according to the manufacturer's protocol and incubated with biotinylated RNAs for $48 \mathrm{~h}$ at room temperature under moderate agitation on a tube rotator. Next, $200 \mu$ l lysed cells were collected and incubated with $400 \mu$ l Streptavidin 

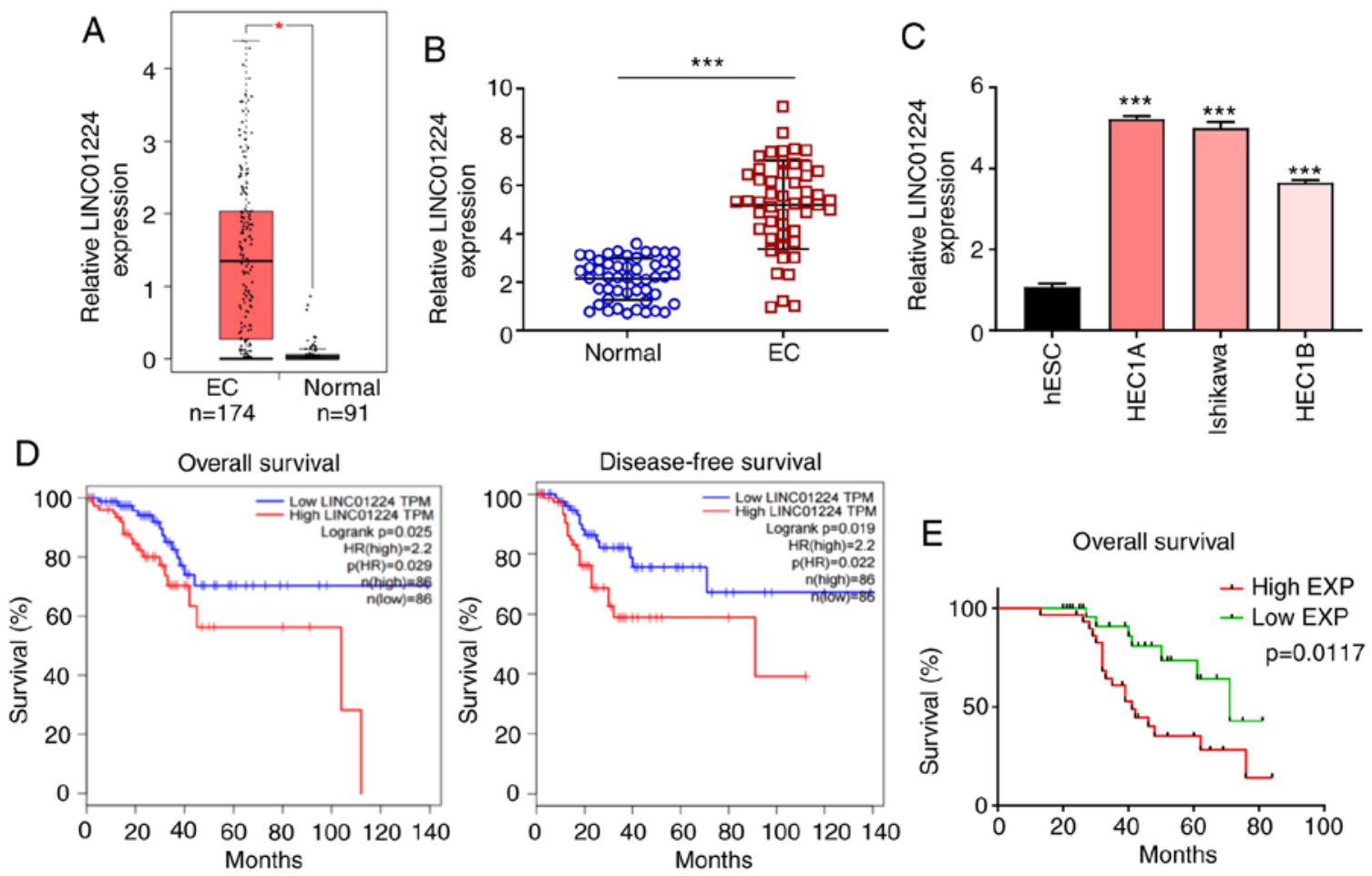

Figure 1. LINC01224 is significantly increased in EC and associated with poor survival rate of patients with EC. (A) Relative LINC01224 mRNA expression levels from TCGA and The Genotype Tissue Expression projects were used to assess LINC01224 expression in EC using GEPIA2. (B) Relative mRNA expression levels of LINC01224 in 50 pairs of EC and adjacent normal tissue were assessed using reverse transcription-quantitative PCR. (C) Relative mRNA expression level of LINC01224 in a human normal ESC and EC cell lines (HEC1A, HEC1B and Ishikawa). Kaplan-Meier analysis was performed to evaluate the association between expression levels of LINC01224 and overall/disease-free survival of patients with EC from (D) TCGA using GEPIA2 and (E) 50 patients with EC with high or low LINC01224 expression (both $\mathrm{n}=25$ ). All data are representative of three independent experiments and presented as the mean \pm SD ${ }^{*} \mathrm{P}<0.05 ;{ }^{* * * *} \mathrm{P}<0.001$ vs. hESCs. EXP, expression; EC, endometrial carcinoma; TCGA, The Cancer Genome Atlas; hESC, human endometrial stromal cell.

agarose beads (Invitrogen; Thermo Fisher Scientific, Inc.) for $1 \mathrm{~h}$ at $37^{\circ} \mathrm{C}$ under moderate agitation on a tube rotator at room temperature. After beads were washed with wash buffer (Invitrogen; Thermo Fisher Scientific, Inc.), the bound RNAs were analyzed by RT-qPCR assay.

Statistical analysis. Data are presented as the mean \pm SD of three independent biological experiments. Statistical analysis was performed using Microsoft Office Excel 2016 (Microsoft Corporation). Significance of differences between two groups was calculated with Student's unpaired t-test. One-way ANOVA followed by Tukey's post hoc test was used to determine the significance among multiple groups. Overall survival was calculated by Kaplan-Meier and log-rank test. Pearson's correlation analysis was performed to assess correlation between RNA expression levels. $\mathrm{P}<0.05$ was considered to indicate a statistically significant difference.

\section{Results}

LINC01224 is significantly increased in EC and associated with poor survival rate of patients with $E C$. RNA sequencing data of LINC01224 expression levels from The Cancer Genome Atlas (TCGA) and the Genotype Tissue Expression (GTEx) projects (gepia.cancer-pku.cn/) was used to evaluate LINC01224 expression in EC using GEPIA2. The data showed that LINC01224 expression was higher in EC tissue $(n=174)$ than in normal endometrial tissue ( $n=91$; Fig. 1A). LINC01224 expression was assessed in 50 pairs of EC clinical samples by RT-qPCR. LINC01224 expression was significantly upregulated in EC tissue compared with matched adjacent normal tissue (Fig. 1B), which was consistent with expression levels in three EC cell lines (HEC1A, HEC1B and Ishikawa) compared with human normal ESCs (Fig. 1C). In the TCGA dataset, patients with EC with high LINC01224 expression had shorter overall and disease-free survival compared with patients with low LINC01224 expression (Fig. 1D). The median LINC01224 expression in EC tissue (Fig. 1B) was selected as the cut-off value (4.813) to divide patients into LINC01224 low- $(n=25)$ and high-expression groups $(n=25)$. Kaplan-Meier curve of overall survival for patients with EC demonstrated that high expression levels of LINC01224 was associated with poor prognosis (Fig. 1E). These results indicated that LINC01224 exerted a tumor-promoting effect on EC progression.

LINC01224 promotes proliferation and inhibits apoptosis of EC cells. To investigate the functional role of LINC01224 in EC, loss of function experiments were performed using HEC1A and Ishikawa cell lines with high LINC01244 expression. Firstly, LINC01224 was knocked down in HEC1A and Ishikawa cell lines using shRNA (Fig. 2A). CCK-8 assay results demonstrated that knockdown of LINC01224 (shLINC01224\#1 and shLINC01224\#2) decreased proliferation of HEC1A and Ishikawa cells in a time-dependent manner (Fig. 2B); this was only significant at $96 \mathrm{~h}$. Colony formation assay indicated that LINC01224 knockdown 

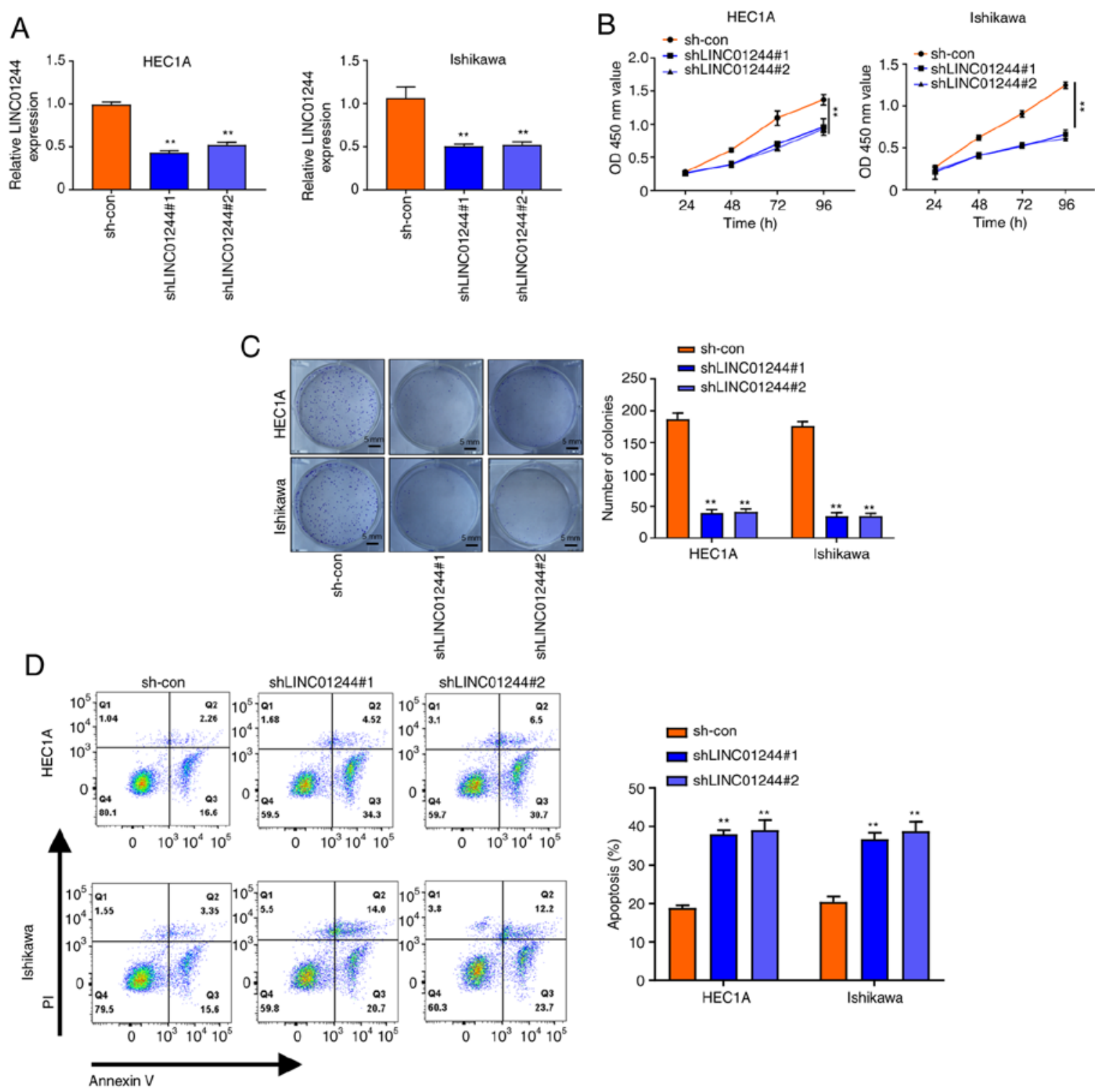

\section{$\mathrm{E}$}
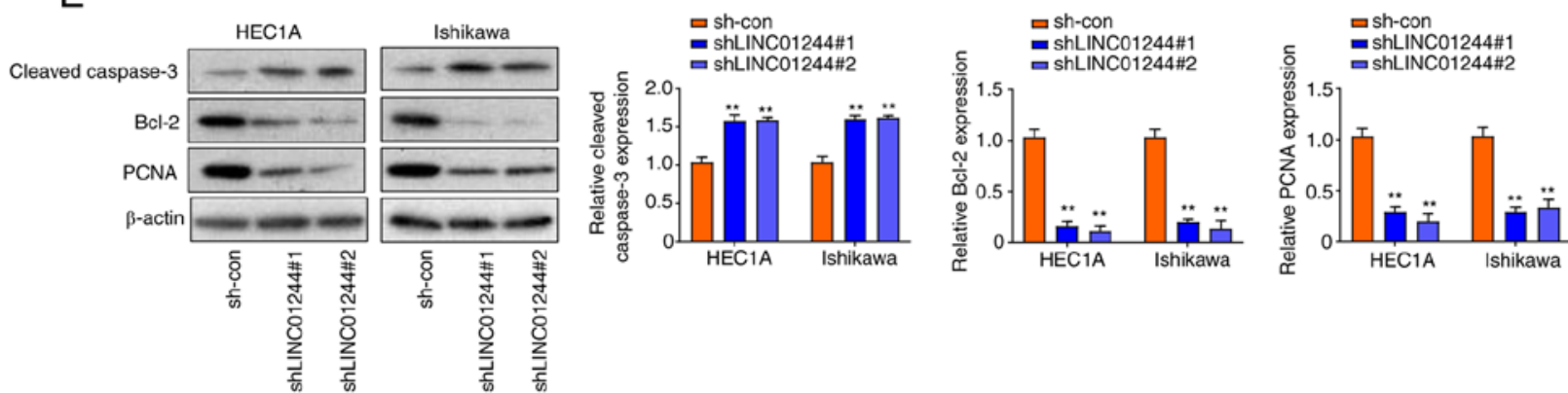

Figure 2. Knockdown of LINC01224 inhibits proliferation and increases apoptosis of EC cells. (A) Reverse transcription-quantitative PCR analysis of LINC01224 expression in HEC1A and Ishikawa cells transfected with sh-con, shLINC01224\#1 or shLINC01224\#2. (B) Cell Counting Kit-8 and (C) colony formation assays were used to assess proliferation of HEC1A and Ishikawa cells transfected with sh-con, shLINC01224\#1 or shLINC01224\#2. (D) Cell apoptosis was determined by Annexin V/PI staining using flow cytometry analysis in HEC1A and Ishikawa cells transfected with sh-con, shLINC01224\#1 or shLINC01224\#2. (E) Representative western blots and quantification of proliferation and apoptosis-associated protein expression levels in HEC1A and Ishikawa cells transfected with sh-con, shLINC01224\#1 or shLINC01224\#2. $\beta$-actin was used as the loading control. All data is representative of three independent experiments and expressed as the mean $\pm \mathrm{SD}$. ${ }^{* *} \mathrm{P}<0.01 \mathrm{vs.} \mathrm{sh-con.} \mathrm{EC,} \mathrm{endometrial} \mathrm{carcinoma;} \mathrm{sh,} \mathrm{short} \mathrm{hairpin;} \mathrm{con,} \mathrm{control;} \mathrm{OD,} \mathrm{optical} \mathrm{density;}$ PCNA, proliferating cell nuclear antigen.

decreased the number of colonies of HEC1A and Ishikawa cells (Fig. 2C). Moreover, flow cytometry assay showed that apoptosis of HEC1A and Ishikawa cells transfected with
shLINC01224 significantly increased compared with the sh-con group (Fig. 2D). Western blotting results showed that knockdown of LINC01224 increased the protein levels of 
A
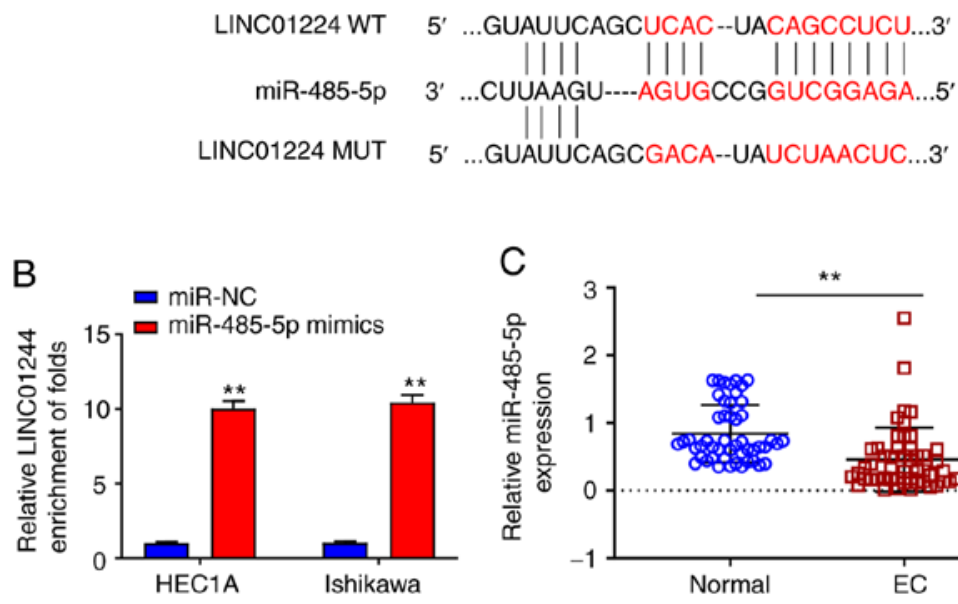

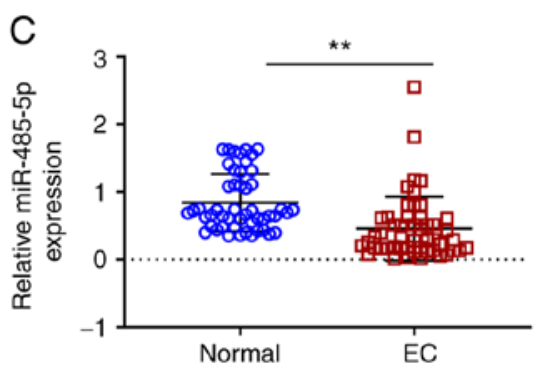

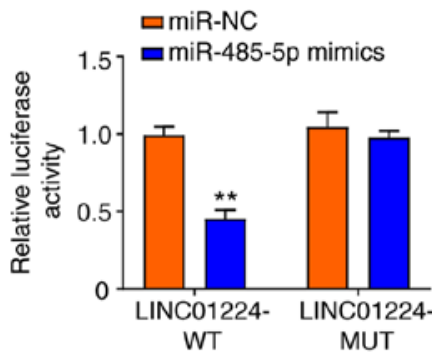

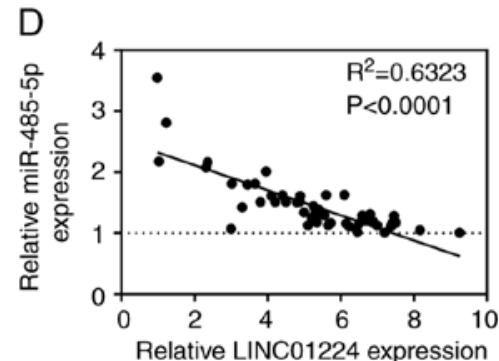

Figure 3. LINC01224 directly binds and negatively regulates expression of miR-485-5p. (A) Predicted binding site of miR-485-5p within LINC01224 using Starbase 3.0. Luciferase activity was determined in HEC1A and Ishikawa cells transfected with miR-485-5p mimics or miR-NC. Binding sequences in LINC01224 WT were mutated to generate LINC01224 MT. (B) RNA pull-down assay was used to determine the interaction between LINC01224 and miR-485-5p. (C) Relative mRNA expression of miR-485-5p in EC and adjacent normal tissue was assessed using reverse-transcription-quantitative PCR. (D) Pearson's correlation analysis between LINC01224 and miR-485-5p expressions in 50 pairs of EC tissue. All data are representative of three independent experiments and presented as the mean $\pm \mathrm{SD} .{ }^{* *} \mathrm{P}<0.01$ vs. miR-NC. miR, microRNA; NC, negative control; WT, wild-type; MT, mutant; EC, endometrial carcinoma.

cleaved caspase- 3 but decreased those of Bcl-2 and PCNA (Fig. 2E). Moreover, overexpression of LINC01224 promoted proliferation and colony formation of HEC1A and Ishikawa cells (Fig. S1A-C). To validate the in vivo effect of LINC01224 in EC progression, a xenograft model of EC was established by subcutaneously injecting LINC01224-knockdown (shLINC01224\#1 and shLINC01224\#2) and sh-con lentiviral-transfected stable HEC1A cells into mice (Fig. S1D). Tumor sizes were measured every 7 days. The results showed that knockdown of LINC01224 decreased both tumor size and weight (Fig. S2A-C). Consistent with cellular assay results, LINC01224 downregulation decreased Bcl-2 and PCNA expression and increased cleaved caspase-3 expression in the HEC1A xenograft tumors (Fig. S2A-D). Collectively, these data suggested that knockdown of LINC01224 inhibited proliferation and apoptosis of EC cells.

LINC01224 directly binds to and negatively regulates expression of miR-485-5p. Accumulating evidence suggested that lncRNAs serve as miRNA sponges to inhibit expression and regulate the biofunction of miRNAs $(6,16)$. Thus, the potential binding miRNA partner of LINC01224 was analyzed using Starbase (data not shown). Results showed that LINC01224 potentially bound to miR-485-5p. Luciferase reporter assay demonstrated that overexpression of miR-485-5p significantly repressed the luciferase activity of LINC01224 WT but not LINC01224 MT (Fig. 3A). RNA pull-down assay indicated that the LINC01224 expression was more enriched on the miR-485-5p probe (Fig. 3B). In 50 pairs of samples from patients with EC, miR-485-5p was significantly downregulated in EC tumor tissue (Fig. 3C), and there was a strong negative correlation between LINC01224 and miR-485-5p (Fig. 3D; P<0.0001). These results demonstrated that
LINC01224 directly interacted with, and inhibited expression of, miR-485-5p.

LINC01224 promotes proliferation and inhibits apoptosis of EC cells via sponging miR-485-5p. To investigate the role of LINC01224 in EC progression, LINC01224 was knocked down in HEC1A and Ishikawa cells (Fig. 4A) and miR-485-5p expression levels were assessed. Knockdown of LINC01224 significantly increased the expression of miR-485-5p; this was partially rescued by treatment with miR-485-5p inhibitor (Figs. 4A and S1F). Based on the aforementioned results, it was hypothesized that the LINC01224/miR-485-5p axis is linked to EC progression. CCK-8 and colony formation assays showed that LINC01224 knockdown inhibited the proliferation of HEC1A and Ishikawa cells but miR-485-5p inhibitor treatment partially reversed this effect (Fig. 4B and C). Flow cytometry assay displayed a similar result for EC cell apoptosis (Fig. 4D), consistent with western blotting results demonstrating that miR-485-5p inhibition restored the increased protein levels of cleaved caspase- 3 and decreased protein levels of Bcl-2 and PCNA in EC cells transfected with shLINC01224 (Fig. S3). Taken together, these results demonstrated that LINC01224 promoted proliferation and inhibited apoptosis of EC cells via sponging miR-485-5p.

LINC01224 elevates AKT3 expression via sponging $m i R-485-5 p$ in EC cells. Previous research has demonstrated that AKT3 is a potential target of miRNA $(19,20)$. It has been reported that lncRNAs serve as competing endogenous RNAs that interact with miRNAs to regulate the expression of key oncogenes (21), involved in tumorigenesis (21). The present study demonstrated that miR-485-5p bound to LINC01224. Starbase 3.0 predicted that miR-485-5p targets the 3'-UTR of 


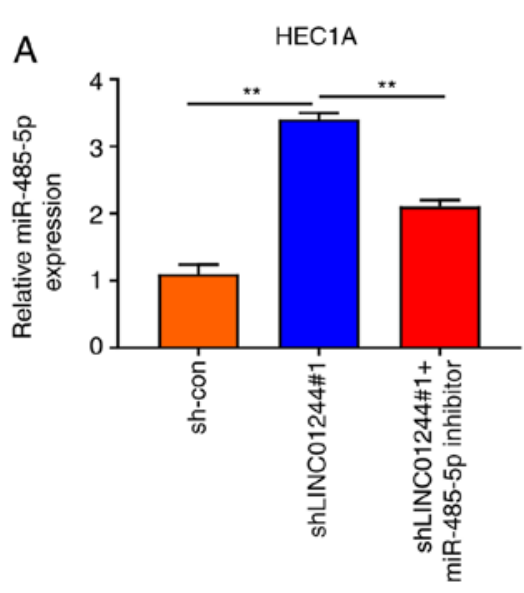

B

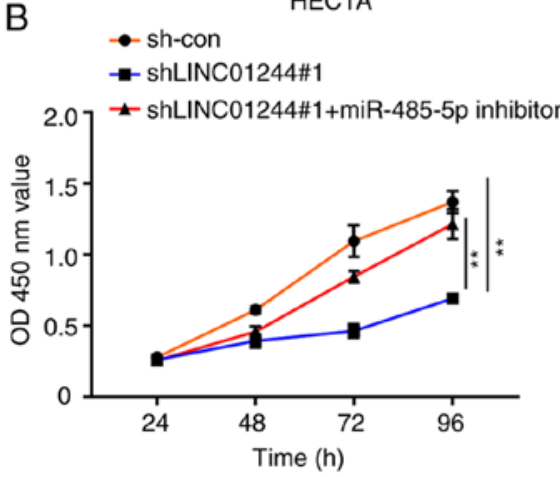

C

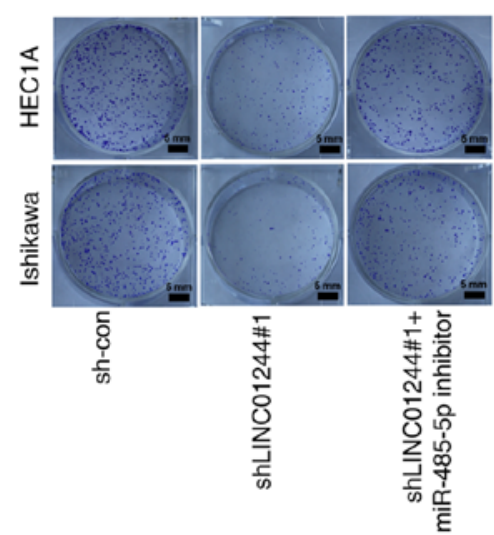

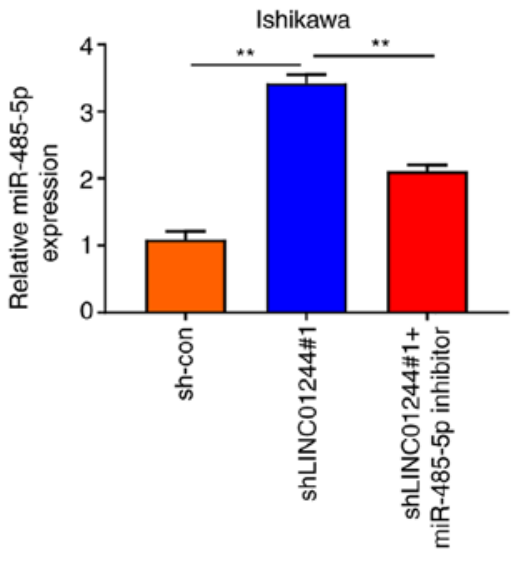

Ishikawa
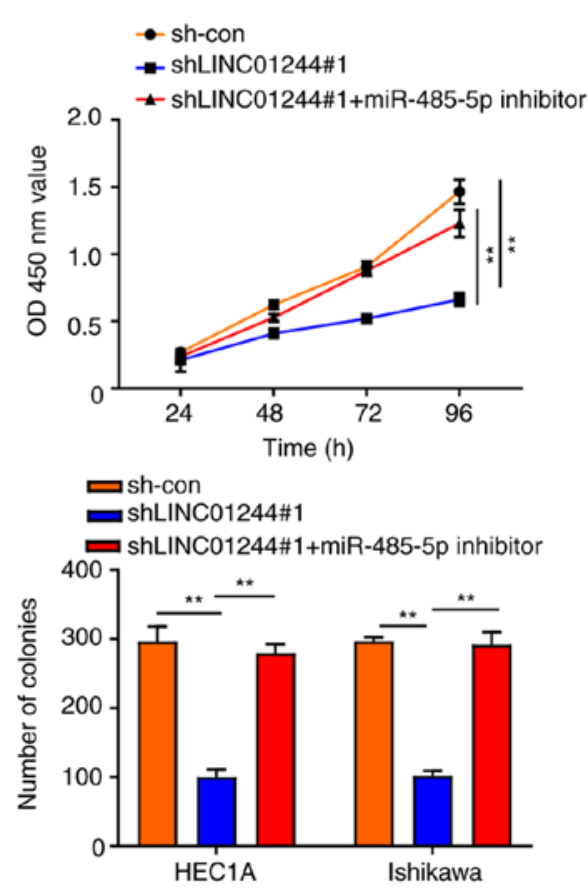

D
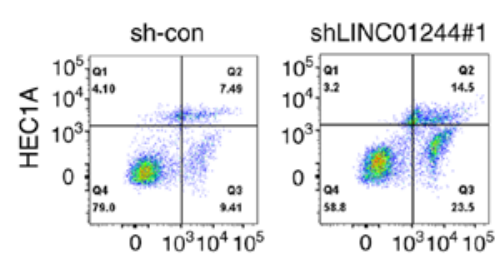

shLINC01244\#1+miR-
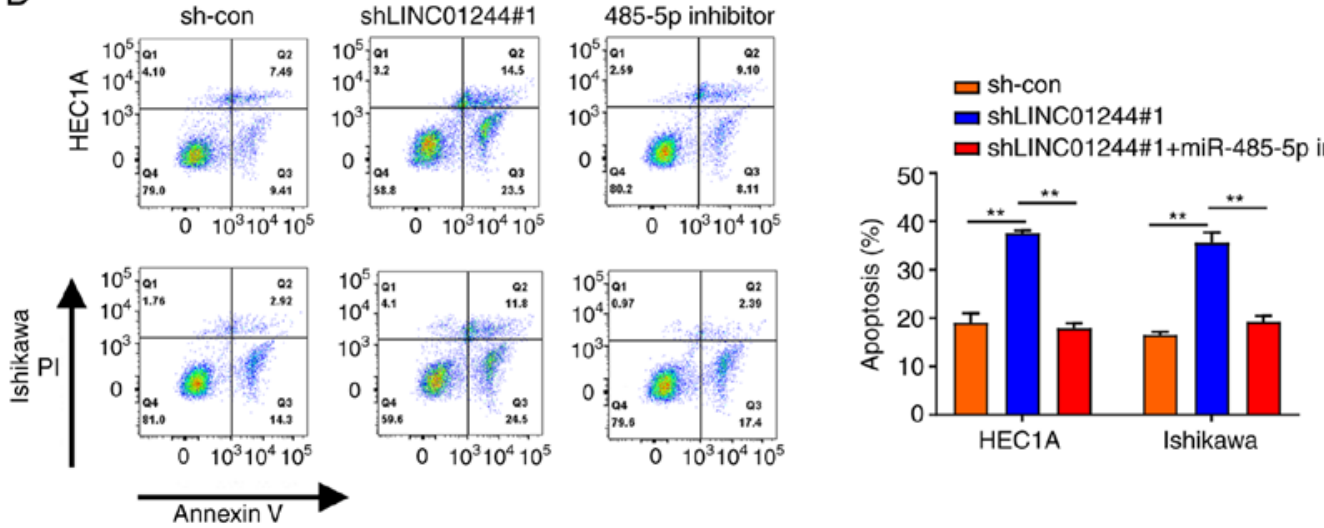

Figure 4. LINC01224 promotes proliferation and inhibits apoptosis of endometrial carcinoma cells via sponging miR-485-5p. (A) Reverse transcription-quantitative PCR analysis was performed to determine miR-485-5p expression in HEC1A and Ishikawa cells transfected with sh-con, shLINC01224\#1 or shLINC01224\#1 + miR-485-5p inhibitor. (B) Cell Counting Kit-8 and (C) colony formation assays were used to measure the proliferation of HEC1A and Ishikawa cells transfected with sh-con, shLINC01224\#1 or shLINC01224\#1 + miR-485-5p inhibitor. (D) Cell apoptosis was determined by Annexin V/PI staining using flow cytometry analysis. Data are representative of three independent experiments and shown as the mean $\pm \mathrm{SD}$. ${ }^{* *} \mathrm{P}<0.01$. miR, microRNA; sh, short hairpin; con, control; OD, optical density. 
A
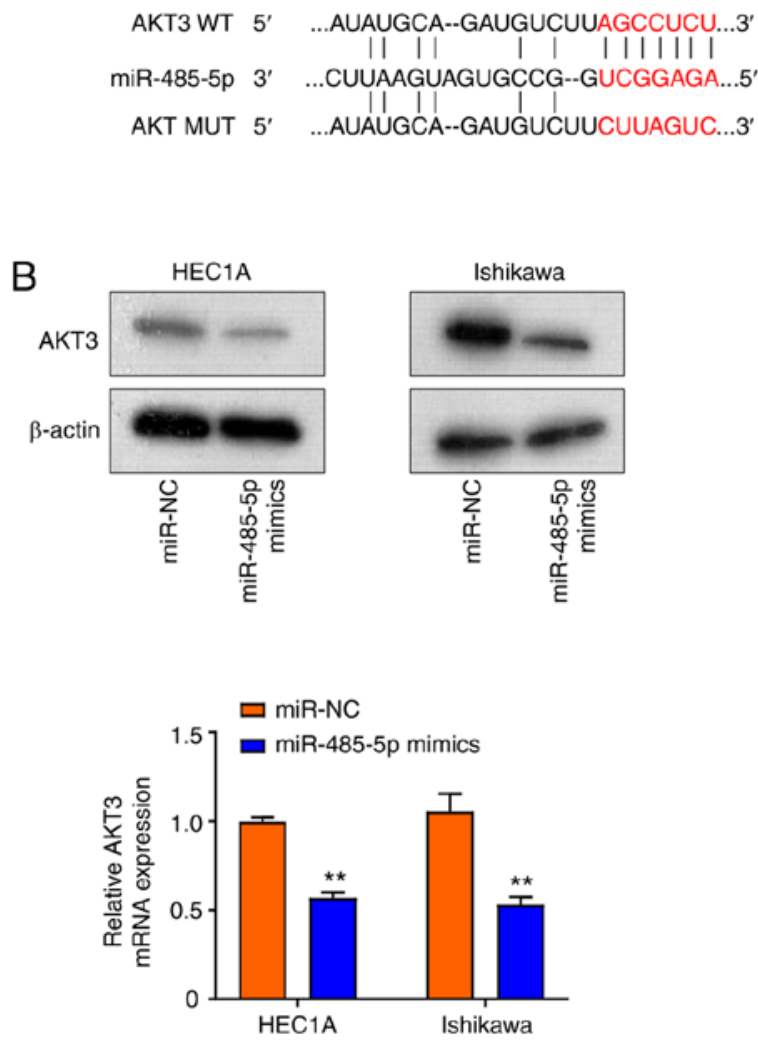
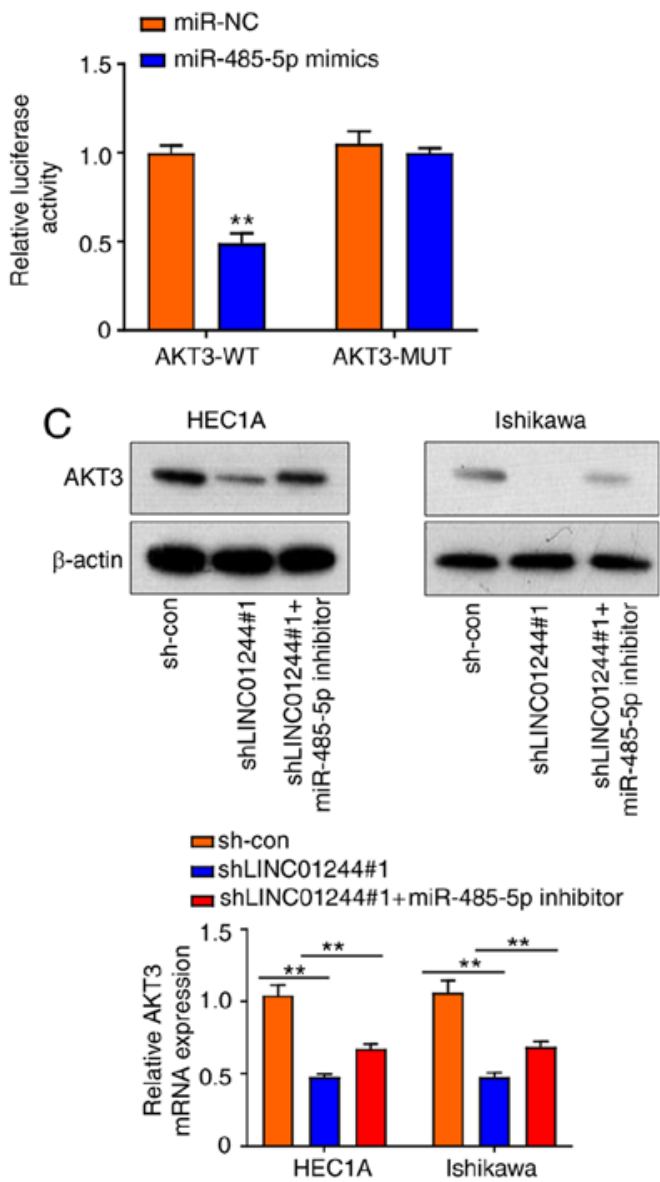

Figure 5. LINC01224 elevates AKT3 expression via sponging miR-485-5p in endometrial carcinoma cells. (A) Predicted binding site of miR-485-5p within AKT3 using Starbase 3.0. Luciferase activity was determined in HEC1A and Ishikawa cells transfected with miR-485-5p mimics or miR-NC. Binding sequences in AKT3 WT were mutated to generate AKT MT. (B) Western blot and reverse transcription-quantitative PCR analysis were performed to determine AKT3 expression in HEC1A and Ishikawa cells transfected with (B) miR-NC or miR-485-5p mimics and (C) sh-con, shLINC01224\#1 or shLINC01224\#1 + miR-485-5p inhibitor. Data are presented as the mean of three independent experiments and shown as the mean \pm SD. ${ }^{* *} \mathrm{P}<0.01 \mathrm{vs}$. miR-NC. miR, microRNA; NC, negative control; MT, mutant; WT, wild-type; sh, short hairpin; con, control.

the AKT3 gene (Fig. 5A). Thus, AKT3 was selected for subsequent analysis. Luciferase reporter assay demonstrated that miR-485-5p mimics significantly inhibited luciferase activity of AKT3 WT; this effect was diminished following mutation of the predicted binding site for miR-485-5p (Fig. 5A), confirming the physical interaction of AKT3 with miR-485-5p. Both the mRNA and protein levels of AKT3 in HEC1A and Ishikawa cells were downregulated by overexpression of miR-485-5p mimics (Fig. 5B). Furthermore, knockdown of LINC01244 significantly decreased AKT3 protein and mRNA levels, which were effectively restored by miR-485-5p inhibitor (Fig. 5C). Collectively, these results indicated that LINC01224 elevated AKT3 expression via sponging miR-485-5p in EC cells.

\section{Discussion}

$\mathrm{EC}$ is the most common cancer in women, but overall clinical outcomes are still unsatisfactory due to the lack of effective therapeutic targets (1-3). Increasing evidence has demonstrated that IncRNAs function as endogenous miRNA sponges by binding to miRNAs and regulating their function, thus participating in tumor initiation and progression, thus presenting potential therapeutic targets and prognostic biomarkers for cancer $(6,9,10,16,22)$. A novel IncRNA, LINC01224, has been discovered to exhibit a tumor-promoting effect in cancer (including hepatocellular carcinoma and ovarian cancer) via miRNA-mediated target gene expression $(12,13)$. However, its clinical significance and underlying functional role of LINC01224 in EC remain unclear. The present study demonstrated that expression of LINC01224 was significantly upregulated in EC tissue and cell lines; this was associated with shorter survival time of patients with EC. Knockdown of LINC01224 impaired EC cell proliferation but promoted apoptosis. The nude mouse xenograft model of LINC01224 knockdown further revealed that LINC01224 increased EC tumor growth in vivo. These results suggested that LINC01224 functioned as an oncogene to promote EC progression.

The involvement of miRNAs in cancer and their significance as clinical biomarkers is increasingly appreciated $(14,23)$. miR-485-5p has been recently found to be downregulated in cancer, including cholangiocarcinoma, hepatocellular carcinoma and breast and lung cancer, and its low expression is positively associated with risk and poor prognosis of cancer, implying that miR-485-5p serves as a tumor suppressor (24-28). AKT3 has been found to mediate resistance to apoptosis in B-RAF-targeted melanoma cells (29). In the present study, bioinformatics analysis (Starbase 3.0) predicted that miR-485-5p was the only downstream miRNA 
target of LINC01224. RNA pull-down and luciferase assays further verified their direct interaction in EC. Additionally, AKT3 was shown to be a direct target of miR-485-5p in EC cells. Functionally, LINC01224 inhibition significantly suppressed EC cell proliferation but increased apoptosis via sponging miR-485-5p; these effects were partially abrogated by miR-485-5p inhibition. Collectively, the present study determined the expression levels, clinical implication and functional mechanism of LINC01224 in EC and revealed that LINC01224 facilitated EC progression via mediating the miR-485-5p/AKT3 axis, thus offering a potential diagnostic biomarker and therapeutic target for EC treatment.

In summary, LINC01224 served as an oncogenic lncRNA to promote EC cell proliferation and inhibit apoptosis via sponging miR-485-5p to elevate AKT3 expression levels, thus providing a promising therapeutic target for EC treatment.

\section{Acknowledgements}

Not applicable.

\section{Funding}

The present study was supported by the Affiliated Yixing Hospital of Jiangsu University.

\section{Availability of data and materials}

The datasets used and/or analyzed during the current study are available from the corresponding author on reasonable request.

\section{Authors' contributions}

$\mathrm{XZ}, \mathrm{WL}, \mathrm{XY}$ and $\mathrm{HZ}$ conceived and supervised the project. XZ, WL, XY, TM, YR, MH, HY, HW and HZ performed the biological experiments. XZ, WL, XY and HZ analyzed data and wrote the manuscript. XY and HZ confirm the authenticity of all the raw data. All authors read and approved the final manuscript.

\section{Ethics approval and consent to participate}

The present study was approved by the ethics committee of the Affiliated Yixing Hospital of Jiangsu University (approval no. 2017-00238). All patients provided written informed consent.

\section{Patient consent for publication}

Not applicable.

\section{Competing interests}

The authors declare that they have no competing interests.

\section{References}

1. Torre LA, Bray F, Siegel RL, Ferlay J, Lortet-Tieulent J and Jemal A Global cancer statistics, 2012. CA Cancer J Clin 65: 87-108, 2015.

2. D'Andrilli G, Bovicelli A, Paggi MG and Giordano A: New insights in endometrial carcinogenesis. J Cell Physiol 227: 2842-2846, 2012.
3. Buhtoiarova TN, Brenner CA and Singh M: Endometrial carcinoma: Role of current and emerging biomarkers in resolving persistent clinical dilemmas. Am J Clin Pathol 145: 8-21, 2016.

4. Lorenzi L, Avila Cobos F, Decock A, Everaert C, Helsmoortel H, Lefever S, Verboom K, Volders PJ, Speleman F, Vandesompele J and Mestdagh P: Long noncoding RNA expression profiling in cancer: Challenges and opportunities. Genes Chromosomes Cancer 58: 191-199, 2019.

5. Derrien T, Johnson R, Bussotti G, Tanzer A, Djebali S, Tilgner H, Guernec G, Martin D, Merkel A, Knowles DG, et al: The GENCODE v7 catalog of human long noncoding RNAs: Analysis of their gene structure, evolution, and expression. Genome Res 22: 1775-1789, 2012.

6. Geisler S and Coller J: RNA in unexpected places: Long non-coding RNA functions in diverse cellular contexts. Nat Rev Mol Cell Biol 14: 699-712, 2013.

7. Khalil AM, Guttman M, Huarte M, Garber M, Raj A, Rivea Morales D, Thomas K, Presser A, Bernstein BE, van Oudenaarden A, et al: Many human large intergenic noncoding RNAs associate with chromatin-modifying complexes and affect gene expression. Proc Natl Acad Sci USA 106: 11667-11672, 2009.

8. DiStefano JK: The emerging role of long noncoding RNAs in human disease. In: Disease Gene Identification: Methods and Protocols. DiStefano JK (ed). Springer, New York, NY, pp91-110, 2018.

9. Hu G, Niu F, Humburg BA, Liao K, Bendi S, Callen S, Fox HS and Buch S: Molecular mechanisms of long noncoding RNAs and their role in disease pathogenesis. Oncotarget 9: 18648-18663, 2018.

10. Kondo Y, Shinjo K and Katsushima K: Long non-coding RNAs as an epigenetic regulator in human cancers. Cancer Sci 108: 1927-1933, 2017.

11. Haemmerle M and Gutschner T: Long non-coding RNAs in cancer and development: Where do we go from here? Int J Mol Sci 16: 1395-1405, 2015

12. Gong D, Feng PC, Ke XF, Kuang HL, Pan LL, Ye Q and Wu JB: Silencing long non-coding RNA LINC01224 inhibits hepatocellular carcinoma progression via microRNA-330-5p-induced inhibition of CHEK1. Mol Ther Nucleic Acids 19: 482-497, 2020.

13. Xing S, Zhang $\mathrm{Y}$ and Zhang J: LINC01224 exhibits cancer-promoting activity in epithelial ovarian cancer through microRNA-485-5p-mediated PAK4 upregulation. Onco Targets Ther 13: 5643-5655, 2020.

14. Bartel DP: MicroRNAs: Genomics, biogenesis, mechanism, and function. Cell 116: 281-297, 2004.

15. Mishra S, Yadav T and Rani V: Exploring miRNA based approaches in cancer diagnostics and therapeutics. Crit Rev Oncol Hematol 98: 12-23, 2016.

16. Hansen TB, Jensen TI, Clausen BH, Bramsen JB, Finsen B, Damgaard CK and Kjems J: Natural RNA circles function as efficient microRNA sponges. Nature 495: 384-388, 2013.

17. Smith CJ and Osborn AM: Advantages and limitations of quantitative PCR (Q-PCR)-based approaches in microbial ecology. FEMS Microbiol Ecol 67: 6-20,2009.

18. Pritt SL and Smith TM: Institutional animal care and use committee postapproval monitoring programs: A proposed comprehensive classification scheme. J Am Assoc Lab Anim Sci 59: 127-131, 2020.

19. Li L and Ma L: Upregulation of miR-582-5p regulates cell proliferation and apoptosis by targeting AKT3 in human endometrial carcinoma. Saudi J Biol Sci 25: 965-970, 2018.

20. Fang Y, Liang X, Xu J and Cai X: miR-424 targets AKT3 and PSAT1 and has a tumor-suppressive role in human colorectal cancer. Cancer Manag Res 10: 6537-6547, 2018.

21. Wang Y, Hou J, He D, Sun M, Zhang P, Yu Y and Chen Y: The emerging function and mechanism of ceRNAs in cancer. Trends Genet 32: 211-224, 2016.

22. Wang KC and Chang HY: Molecular mechanisms of long noncoding RNAs. Mol Cell 43: 904-914, 2011.

23. Adams BD, Kasinski AL and Slack FJ: Aberrant regulation and function of microRNAs in cancer. Curr Biol 24: R762-R776, 2014.

24. Bao W, Cao F, Ni S, Yang J, Li H, Su Z and Zhao B: lncRNA FLVCR1-AS1 regulates cell proliferation, migration and invasion by sponging miR-485-5p in human cholangiocarcinoma. Oncol Lett 18: 2240-2247, 2019.

25. Tu J, Zhao Z, Xu M, Chen M, Weng Q and Ji J: LINC00460 promotes hepatocellular carcinoma development through sponging miR-485-5p to up-regulate PAK1. Biomed Pharmacother 118: 109213, 2019. 
26. Peng Y, Leng W, Duan S and Hong M: Long noncoding RNA BLACAT1 is overexpressed in hepatocellular carcinoma and its downregulation suppressed cancer cell development through endogenously competing against hsa-miR-485-5p. Biomed Pharmacother 116: 109027, 2019.

27. Lu H, Wang C, Xue L, Zhang Q, Luh F, Wang J, Lin TG, Yen Y and Liu X: Human mitotic centromere-associated kinesin is targeted by MicroRNA 485-5p/181c and prognosticates poor survivability of breast cancer. J Oncol 2019: 2316237, 2019.
28. Gao F, Wu H, Wang R, Guo Y, Zhang Z, Wang T, Zhang G, Liu C and Liu J: MicroRNA-485-5p suppresses the proliferation, migration and invasion of small cell lung cancer cells by targeting flotillin-2. Bioengineered 10: 1-12, 2019.

29. Shao Y and Aplin AE: Akt3-mediated resistance to apoptosis in B-RAF-targeted melanoma cells. Cancer Res 70: 6670-6681, 2010.

(i) (2) This work is licensed under a Creative Commons Attribution-NonCommercial-NoDerivatives 4.0 International (CC BY-NC-ND 4.0) License. 